\title{
A Modified Artificial Bee Colony Algorithm for $p$-Center Problems
}

\author{
Alkın Yurtkuran and Erdal Emel \\ Department of Industrial Engineering, Uludag University, Görükle Campus, 16059 Bursa, Turkey \\ Correspondence should be addressed to Alkın Yurtkuran; alkin@uludag.edu.tr
}

Received 31 August 2013; Accepted 11 November 2013; Published 29 January 2014

Academic Editors: A. G. Hernández-Díaz and W. Szeto

Copyright (C) 2014 A. Yurtkuran and E. Emel. This is an open access article distributed under the Creative Commons Attribution License, which permits unrestricted use, distribution, and reproduction in any medium, provided the original work is properly cited.

\begin{abstract}
The objective of the $p$-center problem is to locate $p$-centers on a network such that the maximum of the distances from each node to its nearest center is minimized. The artificial bee colony algorithm is a swarm-based meta-heuristic algorithm that mimics the foraging behavior of honey bee colonies. This study proposes a modified $\mathrm{ABC}$ algorithm that benefits from a variety of search strategies to balance exploration and exploitation. Moreover, random key-based coding schemes are used to solve the $p$-center problem effectively. The proposed algorithm is compared to state-of-the-art techniques using different benchmark problems, and computational results reveal that the proposed approach is very efficient.
\end{abstract}

\section{Introduction}

The $p$-center problem, which is also known as the minimax location-allocation problem, is one of the best-known NPhard problems in the field of logistics and discrete facility location [1]. This problem consists of locating $p$ facilities (centers) on a network such that the maximum of the distances between nodes and their nearest centers is minimized. In the $p$-center problem, $n$ nodes (customers) and distances between nodes are given, and centers should be located at any given node. The $p$-center problem can be used in a variety of real-life applications such as locating fire stations, police departments, or emergency centers.

The $p$-center problem is NP hard [2], and the number of feasible solutions is $\left(\begin{array}{c}N \\ C\end{array}\right)$, where $N$ is the node number and $C$ is the number of centers. Various heuristics have been proposed to solve the $p$-center problem [3-5]. Moreover, exact algorithms that can solve the problem in certain conditions have been proposed $[4,6,7]$.

The $p$-center problem has attracted increasing attention in recent years. D. Chen and R. Chen [1] proposed new relaxation techniques for the solution of $p$-center problems. Mladenović et al. [8] presented a basic variable neighborhood search and two tabu search heuristics for the $p$-center problem without triangle inequality. Caruso et al. [9] proposed a metaheuristic algorithm, called Dominant, in which a series of set-covering problems according to a predefined maximum distance are solved. Pacheco and Casado [10] proposed a new scatter search-based approach for the $p$ center problem. Davidović et al. [11] introduced an improved bee colony algorithm (BCO), proposing a new concept based on improving the complete solution held by each bee, instead of applying constructive BCO; moreover, they showed that the proposed algorithm produces high-quality solutions within negligible CPU times.

The artificial bee colony ( $\mathrm{ABC}$ ) algorithm, which is a biologically inspired population-based metaheuristic algorithm, was recently introduced for continuous function optimization by Karaboga [12]. Due to its simplicity and ease of implementation, the $\mathrm{ABC}$ algorithm has been extensively applied to both continuous and discrete optimization problems since its invention. Various comparison studies, in which the $\mathrm{ABC}$ algorithm was compared to novel metaheuristic algorithms, such as particle swarm optimization (PSO), differential evolution (DE), and genetic algorithm (GA), have been performed to show its effectiveness $[13,14]$. These studies show that the $\mathrm{ABC}$ algorithm outperforms other novel algorithms on several instance problems.

However, several studies have shown that the $A B C$ algorithm is good at exploration but poor at exploitation. 
The local search equation, in which a new neighbor solution is generated, has received extensive attention because it controls the exploration-exploitation balance. Therefore, to improve the performance of the algorithm, various modifications to the search equation have been proposed in the literature. Zhu and Kwong [15] proposed a global best guided ABC algorithm, which additionally uses the global best information in the search equation as in PSO. Inspired by DE, Gao and Liu introduced a modified version of the $\mathrm{ABC}$ algorithm in which $\mathrm{ABC} / \mathrm{Best} / 1$ and $\mathrm{ABC} / \mathrm{Rand} / 1$ were employed as local search equations [16, 17]. Li et al. [18] presented a modified version of $A B C$ (IABC) in which the best-so-far information, inertial weight, and acceleration coefficient are used when employing local search. Furthermore, Kang et al. [19] proposed the Rosenbrock ABC algorithm, which combines Rosenbrock's rotational method and the original ABC algorithm. Singh [20] used the ABC algorithm to solve the leaf-constrained minimum spanning tree problem. To improve exploration, Alatas [21] employed chaotic maps as initialization and chaotic searches as a local search equation. Gao et al. [22] proposed a new search equation and used Powell's method as a local search technique. Xu et al. [23] described a new $\mathrm{ABC}(\mathrm{NABC})$ algorithm that employs a modified version of the DE/Best/1 strategy. Akay and Karaboga [24] proposed a modified version of the ABC algorithm in which a control parameter that determines the number of parameters to be modified during the production of a neighboring solution is introduced. Moreover, an adaptive technique for reduction of step size was also used.

Although the ABC algorithm was originally developed for solving continuous optimization problems, the use of the $\mathrm{ABC}$ algorithm for combinatorial problems has attracted extensive attention in recent years. Kashan et al. [25] presented DisABC for solving binary optimization problems. In DisABC, a measure of dissimilarity between binary vectors is used instead of vector subtraction, which is used in the original ABC algorithm's search equation. Szeto et al. [26] proposed a modified ABC algorithm for capacitated vehicle routing problems and introduced various operators to produce new neighbor solutions. Uzer et al. [27] used a hybrid ABC-support vector machine mechanism for feature selection and classification for medical databases. AlvaradoIniesta et al. [28] used the $\mathrm{ABC}$ algorithm for the optimization of the material flow in a manufacturing plant. Ji et al. [29] proposed a modified ABC algorithm for solving 0-1 multidimensional knapsack problems and introduced a novel communication mechanism based on the updating and diffusion of inductive pheromones produced by bees. Furthermore, numerous papers have used the ABC algorithm for scheduling problems, such as the lot-streaming flow shop scheduling problem [30], total flow time minimization in permutation flow shops [31], the flexible job-shop scheduling problem [32], and the no-idle permutation flow shop scheduling problem with total tardiness criterion [33]. Readers can refer to Karaboga et al. [34] for an extensive literature review of the $\mathrm{ABC}$ algorithm and its applications.

In this paper, a modified $\mathrm{ABC}$ algorithm (M-ABC) is proposed to solve well-known $p$-center problems. The $\mathrm{M}$ ABC algorithm uses random key-based encoding for solution representation and employs a new multisearch strategy in which different search strategies are used for generating new neighbor solutions. The remainder of this paper is organized as follows: Section 2 gives the mathematical model for the $p$-center problem, Section 3 covers the traditional $\mathrm{ABC}$ algorithm, and the proposed algorithm is described in Section 4. Computational results are given in Section 5, and finally, Section 6 concludes the paper.

\section{Mathematical Formulation}

The $p$-center problem can be briefly defined as follows. Let $N=\{1,2,3, \ldots, n\}$ be the node set, and there is a distance $d_{i j}$ associated with each node pair $(i, j) \in N, i \neq j$. The objective is to locate $p$ centers, $P=\{1,2,3, \ldots, p\}$, at given nodes so that the maximum distance between a node and its nearest facility $p$ is minimized. The $p$-center problem can be mathematically formulated as follows [11].

Decision variables are as follows:

$$
\begin{gathered}
x_{i j}= \begin{cases}1, & \text { if node } i \text { is assigned to center at node } j, \\
0, & \text { otherwise }\end{cases} \\
c_{j}= \begin{cases}1, & \text { if node } j \text { is the center, } \\
0, & \text { otherwise. }\end{cases}
\end{gathered}
$$

Parameters are as follows:

$$
\begin{aligned}
& N \text { : set of nodes, } \\
& d_{i j} \text { : travel time (distance) from node } i \text { to } j \text {, where } i, j \in \\
& N \text {, } \\
& \text { p: number of centers. } \\
& \text { Minimize } \quad \sum_{i \in N} \sum_{j \in N} d_{i j} * x_{i j} \\
& \text { subject to } \quad \sum_{j \in N} x_{i j}=1, \quad \forall i \in N, i \neq j, \\
& \qquad x_{i j} \leq c_{j}, \quad \forall i, j \in N, j \neq i \\
& \qquad \sum_{j \in N} c_{j}=p, \quad \forall j \in N, \\
& \qquad x_{i j}, y_{j} \in\{0,1\} \quad \forall i, j \in N .
\end{aligned}
$$

The objective function represents the minimization of the largest distance between a node and its closest facility. Constraint (3) expresses that each node should be assigned to a center. Constraint (4) prevents any center from being located outside the node set. The total number of located facilities is set to $p$ by constraint (5).

\section{Artificial Bee Colony Algorithm}

The ABC algorithm is a population-based metaheuristics algorithm that mimics the foraging behavior of honey bee swarms. The ABC algorithm classifies bees in a colony 
into three main groups: employed bees, onlooker bees, and scout bees. Employed bees are responsible for exploiting the food sources and sharing the information about these food sources. Onlooker bees wait in the hive and take the food source information from employed bees to make a decision on further exploiting the food source. Scout bees randomly search the environment to find a new food source.

In the $\mathrm{ABC}$ algorithm, each candidate solution to the problem is associated with a food source and is represented by an $n$-dimensional real-coded vector. The quality of a solution corresponds to the nectar amount on that food source, and one employed bee explores each food source. In other words, the number of the employed bees is equal to the number of food sources. The colony is equally divided into employed and onlooker bees. A food source, which cannot be improved for a predetermined number of tries, is abandoned and the employed bee associated with that food source becomes a scout. In the $\mathrm{ABC}$ algorithm, the employed and onlooker bees are responsible for exploiting, whereas the scout bees handle exploring.

The main steps of the ABC algorithm are as follows [12]:

(1) initialization,

(2) evaluating the population,

(3) repeat,

(4) employed bee phase,

(5) onlooker bee phase,

(6) scout bee phase,

(7) until (termination criteria are satisfied).

3.1. Initialization. In the initialization step, the $\mathrm{ABC}$ algorithm generates a randomly distributed population of $\mathrm{SN}$ solutions (food sources), where SN denotes the number of employed or onlooker bees. Let $x_{i}=\left\{x_{i, 1}, x_{i, 2}, \ldots, x_{i, D}\right\}$ represent the $i$ th food source, where $D$ is the problem size. Each food source is generated within the range of the boundaries of the parameters by

$$
x_{i, j}=x_{j}^{\min }+\operatorname{rand}(0,1)\left(x_{j}^{\max }-x_{j}^{\min }\right) \text {, }
$$

where $i=1, \ldots, \mathrm{SN}, j=1, \ldots, D \cdot x_{j}^{\min }$, and $x_{j}^{\max }$ are the lower and upper bound for the dimension $j$, respectively.

3.2. Employed Bee Phase. In the employed bee phase, employed bees generate a neighboring food source $v_{i}$ by performing a local search around each food source $i \in$ $\{1,2, \ldots, \mathrm{SN}\}$ as follows:

$$
v_{i, j}=x_{i, j}+\Phi_{i, j}\left(x_{i, j}-x_{k, j}\right),
$$

where $j$ is a random integer in the range $[1, D]$ and $k \epsilon$ $\{1,2, \ldots, \mathrm{SN}\}$ is randomly chosen food source that is not equal to $i . \Phi_{i, j}$ is a random number in the range $[-1,1]$. A greedy selection is applied between $x_{i}$ and $v_{i}$ in which the better solution will be retained. Then, employed bees will return to their hive and share the information on new solutions with onlooker bees.
3.3. Onlooker Bee Phase. Onlooker bees select a food source depending on the probability value prob associated with that food source. The value $p$ is calculated as follows:

$$
\operatorname{prob}_{i}=\frac{f_{i}}{\sum_{j=1}^{\mathrm{SN}} f_{j}},
$$

where $f_{i}$ is the objective function value of solution $i$. By using this mechanism, food sources having better fitness values will be more likely to be selected. Once the onlooker bee has chosen the food source, she generates a new solution using (7). As in the employed bee phase, a greedy selection is carried out between $x_{i}$ and $v_{i}$.

In the employed bee phase, a local search is applied to every food source, whereas only the selected food sources will be updated in the onlooker bee phase.

3.4. Scout Bee Phase. If a food source cannot be improved for a predetermined number of tries, then the employed bee associated with that food source becomes a scout bee. Then, the scout bee finds a new food source using (8). After the scout bee finds a new source, she becomes an employed bee again.

\section{Proposed Artificial Bee Colony Algorithm}

4.1. Random Key-Based Encoding. Because the ABC algorithm was first introduced for solving continuous optimization problems, real-coded solution vectors can be used directly in the calculation step of the objective function. To apply the $\mathrm{ABC}$ algorithm for combinatorial optimization problems like $p$-center problems, real-coded vectors should be converted to permutations of nodes. Therefore, in the proposed study, random key-based encoding is used to represent solutions.

The random key procedure was first introduced by Bean [35] for solving sequencing problems with genetic algorithms using real-coded genes. Snyder and Daskin [36] have also used random key-based encoding schemes to overcome the infeasibility problems in genetic algorithms. Moreover, numerous studies used random key-based encoding to convert real-coded vectors to feasible solutions [37-41].

In the proposed solution encoding, for any $n$-dimensional problem, when the real-coded solution values are sorted in a nondecreasing order, their corresponding indices (treated as centers) in the sorted order yield a solution. Original to the $p$-center problem, the number of centers is limited by the problem as $p$. Therefore the first $p$ values of the sorted index vector are taken as the solution in this study. Once the center nodes are determined, the objective value is calculated as follows: $\max _{i \in N}\left\{\min _{j \in P}\left\{d\left(i, c_{j}\right)\right\}\right\}$ where $c$ denotes the center nodes, $d$ is the distance operator, $N$ represents nodes set, and $P=(1,2, \ldots, p)$.

An example of this procedure is given in Figure 1 for a problem $(p=5, n=8)$, where first the real-coded food source vector is sorted and then the first $p$ indices of the sorted index vector are chosen as the centers. 


\begin{tabular}{rl|c|c|c|c|c|c|c|c|} 
Index number (IN): & 1 & 2 & 3 & 4 & 5 & 6 & 7 & 8 \\
\cline { 2 - 10 } Food source (FS): & 1,95 & $-2,34$ & 4,43 & 6,32 & 0,32 & $-4,04$ & 3,30 & $-9,05$ \\
\hline
\end{tabular}

FIGURE 1: Example of random key-based encoding.

4.2. Multisearch Strategy. The ABC algorithm has been shown to be good at exploration but poor at exploitation $[15,16]$. Therefore, numerous studies have been performed to improve the performance of the $\mathrm{ABC}$ algorithm, and most of these studies investigated modifications for the search equation because it is the main part of the algorithm that controls the exploration-exploitation balance [15].

Each search strategy has distinctive characteristics and therefore may have an advantage or disadvantage in different stages of the overall search progress on different problems. In the present study, different search strategies taken from the literature are employed in a random manner to overcome this problem. In this paper, a new neighbor solution is generated using a search equation selected randomly from a candidate pool, which consists of selected search strategies. Four search equations are used, which are explained as follows.

(i) Original ABC [12]: described in detail in Section 3.2. The search equation is as follows:

$$
v_{i, j}=x_{i, j}+\Phi_{i, j}\left(x_{i, j}-x_{k, j}\right) .
$$

(ii) Global best guided ABC [15]: inspired by PSO, Zhu and Kwong [15] described a new search equation to improve the exploitation and to take advantage of the global best solution information as follows:

$v_{i, j}=x_{i, j}+\Phi_{i, j}\left(x_{i, j}-x_{k, j}\right)+\Psi_{i, j}\left(y_{j}-x_{i, j}\right)$,

where $y_{j}$ is the $j$ th element of global best solution, $\Psi_{i, j}$ is a uniform random number in $[0, C]$ where $C$ is a nonnegative constant and is suggested to be 1.5 , $\Phi_{i, j}$ is a random number in the range $[-1,1]$, and $j \in\{1,2, \ldots, n\}$ is a randomly chosen index.

(iii) $\mathrm{ABC} / \mathrm{best} / 1$ [16]: based on the differential evolution algorithm, Gao and Liu [16] proposed a modified search equation as follows:

$$
v_{i, j}=x_{\text {best }, j}+\Phi_{i, j}\left(x_{r 1, j}-x_{r 2, j}\right),
$$

where the indices $r 1$ and $r 2$ are mutually exclusive integers randomly chosen from $\{1,2, \ldots, \mathrm{SN}\}$ and different from the base index $i ; x_{\text {best }}$ is the best solution in the current population and $j \in\{1,2, \ldots, n\}$ is a randomly chosen index; and $\Phi_{i, j}$ is a random number in the range $[-1,1]$.

(iv) ABC with Powell's method [22]: in order to improve the search ability and convergence speed of the search equation proposed in [15], Gao et al. [22] described a new search equation that uses the information of a randomly selected solution and the best solution as follows:

$$
v_{i, j}=x_{k, j}+\operatorname{rand}(0,1)\left(x_{\text {best }, j}-x_{k, j}\right),
$$

where $\operatorname{rand}(0,1)$ denotes a uniformly distributed random number, $k$ is an integer uniformly chosen from the range $[1, \mathrm{SN}]$ and is different from $i$, and $x_{\text {best }}$ is the best solution in the current population.

4.3. Proposed Algorithm. Based on the explanations in the previous subsection, the general scheme of the $M-A B C$ is given in Pseudocode 1.

\section{Computational Results}

In this section, the proposed $\mathrm{M}-\mathrm{ABC}$ algorithm, which is discussed in the previous section, is analyzed in detail using several benchmarking problems. The M-ABC algorithm was coded using the $\mathrm{C}++$ language executed on a computer with $2.00 \mathrm{~GB}$ RAM and an Intel Core2 Duo $2.00 \mathrm{GHz} \mathrm{CPU}$. In order to evaluate the validity and success of $\mathrm{M}-\mathrm{ABC}$, the described algorithm is applied to 40 well-known benchmark problems that were originally developed for $p$-median problems [42]. These benchmark problems can be downloaded from ORLibrary at the following website: http://people.brunel.ac.uk/ mastjjb/ jeb/orlib/pmedinfo.html. The problem parameters range from 100 to 900 and from 5 to 200 for the number of customers and the number of centers, respectively.

The proposed $\mathrm{M}-\mathrm{ABC}$ approach is compared with the novel metaheuristic algorithms such as the improved bee colony algorithm (BCOi) [11], multistart interchange (M-I), variable neighborhood search (VNS), tabu search 1 (TS-1) and tabu search 2 (TS-2), proposed in [8], and scatter search (SS), proposed in [10]. To fairly compare the algorithms, 


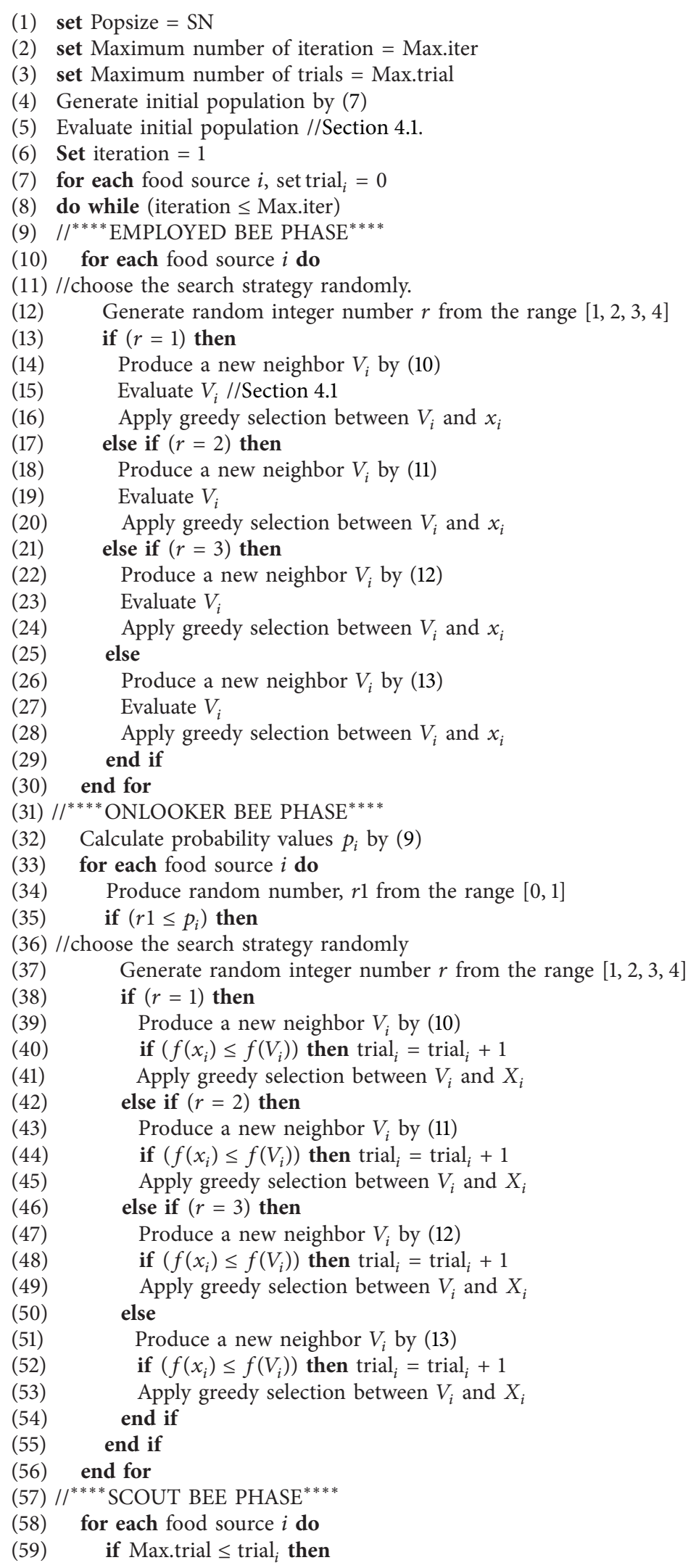


(60) Replace $x_{i}$ with a new randomly generated solution (7)

(61) end if

(62) end for

(63) Save necessary information

(64) iteration $=$ iteration +1

(65) end while

Pseudocode 1: Pseudocode of M-ABC.

TABLE 1: Computational results for benchmark problems.

\begin{tabular}{|c|c|c|c|c|c|c|c|c|c|}
\hline Problem & $n$ & $p$ & M-I & VNS & TS-1 & TS-2 & SS & BCOi & M-ABC \\
\hline Pmed1 & 100 & 5 & 127 & 127 & 127 & 127 & 127 & 127 & 127 \\
\hline Pmed2 & 100 & 10 & 98 & 98 & 98 & 98 & 98 & 98 & 98 \\
\hline Pmed3 & 100 & 10 & 93 & 93 & 93 & 93 & 93 & 93 & 93 \\
\hline Pmed4 & 100 & 20 & 74 & 74 & 74 & 74 & - & 74 & 74 \\
\hline Pmed5 & 100 & 33 & 48 & 48 & 48 & 48 & - & 48 & 48 \\
\hline Pmed6 & 200 & 5 & 84 & 84 & 84 & 84 & 84 & 84 & 84 \\
\hline Pmed7 & 200 & 10 & 64 & 64 & 64 & 64 & 64 & 64 & 64 \\
\hline Pmed8 & 200 & 20 & 58 & 55 & 55 & 55 & - & 55 & 55 \\
\hline Pmed9 & 200 & 40 & 46 & 37 & 37 & 37 & - & 37 & 37 \\
\hline Pmed10 & 200 & 67 & 30 & 20 & 20 & 20 & - & 20 & 20 \\
\hline Pmed11 & 300 & 5 & 59 & 59 & 59 & 59 & 59 & 59 & 59 \\
\hline Pmed12 & 300 & 10 & 51 & 51 & 51 & 51 & 51 & 51 & 51 \\
\hline Pmed13 & 300 & 30 & 41 & 36 & 36 & 36 & - & 37 & 36 \\
\hline Pmed14 & 300 & 60 & 36 & 26 & 26 & 26 & - & 27 & 26 \\
\hline Pmed15 & 300 & 100 & 29 & 18 & 25 & 18 & - & 18 & 18 \\
\hline Pmed16 & 400 & 5 & 47 & 47 & 47 & 47 & 47 & 47 & 47 \\
\hline Pmed17 & 400 & 10 & 40 & 39 & 39 & 39 & 39 & 39 & 39 \\
\hline Pmed18 & 400 & 28 & 29 & 28 & 28 & 35.71 & - & 29 & 30 \\
\hline Pmed19 & 400 & 80 & 28 & 19 & 23 & 19 & - & 19 & 19 \\
\hline Pmed20 & 400 & 133 & 26 & 14 & 22 & 14 & - & 14 & 14 \\
\hline Pmed21 & 500 & 5 & 40 & 40 & 40 & 40 & 40 & 40 & 40 \\
\hline Pmed22 & 500 & 10 & 40 & 38 & 38 & 38 & 38 & 39 & 38 \\
\hline Pmed23 & 500 & 50 & 30 & 23 & 23 & 23 & - & 23 & 23 \\
\hline Pmed24 & 500 & 100 & 25 & 16 & 18 & 16 & - & 16 & 16 \\
\hline Pmed25 & 500 & 167 & 22 & 12 & 24 & 12 & - & 12 & 12 \\
\hline Pmed26 & 600 & 5 & 38 & 38 & 38 & 38 & 38 & 38 & 38 \\
\hline Pmed27 & 600 & 10 & 33 & 32 & 32 & 32 & 32 & 32 & 32 \\
\hline Pmed28 & 600 & 60 & 25 & 19 & 19 & 19 & - & 19 & 19 \\
\hline Pmed29 & 600 & 120 & 23 & 13 & 23 & 13 & - & 14 & 13 \\
\hline Pmed30 & 600 & 200 & 20 & 11 & 19 & 11 & - & 10 & 11 \\
\hline Pmed31 & 700 & 5 & 30 & 30 & 30 & 30 & 30 & 30 & 30 \\
\hline Pmed32 & 700 & 10 & 30 & 29 & 29 & 29 & 29 & 29 & 29 \\
\hline Pmed33 & 700 & 70 & 22 & 16 & 16 & 16 & - & 16 & 16 \\
\hline Pmed34 & 700 & 140 & 21 & 12 & 20 & 12 & - & 12 & 12 \\
\hline Pmed35 & 800 & 5 & 30 & 30 & 30 & 30 & 30 & 30 & 30 \\
\hline Pmed36 & 800 & 10 & 28 & 27 & 27 & 27 & 27 & 28 & 28 \\
\hline Pmed37 & 800 & 80 & 23 & 16 & 22 & 16 & - & 16 & 16 \\
\hline Pmed38 & 900 & 5 & 29 & 29 & 29 & 29 & 29 & 29 & 29 \\
\hline Pmed39 & 900 & 10 & 24 & 24 & 23 & 24 & 23 & 24 & 23 \\
\hline Pmed40 & 900 & 90 & 21 & 14 & 22 & 14 & - & 14 & 14 \\
\hline Best/total & & & $15 / 40$ & $38 / 40$ & $31 / 40$ & $37 / 40$ & $19 / 19$ & $33 / 40$ & $37 / 40$ \\
\hline
\end{tabular}


TABLE 2: CPU time comparison.

\begin{tabular}{|c|c|c|c|c|c|c|c|}
\hline \multirow{2}{*}{ Problem } & \multicolumn{7}{|c|}{ Normalized computational time (sec) } \\
\hline & M-I (90 MFlop) & VNS (90 MFlop) & TS-1 (90 MFlop) & TS-2 (90 MFlop) & SS (50 MFlop) & BCOi (750 MFlop) & M-ABC (500 MFlop) \\
\hline Pmed1 & 0,00 & 0,00 & 0,00 & 0,00 & 0,01 & 0,00 & 0,00 \\
\hline Pmed2 & 1,20 & 0,53 & 0,05 & 0,00 & 0,08 & 0,00 & 0,02 \\
\hline Pmed3 & 3,14 & 0,02 & 1,38 & 1,26 & 0,03 & 0,00 & 0,09 \\
\hline Pmed4 & 14,37 & 0,04 & 0,72 & 0,41 & & 0,00 & 0,56 \\
\hline Pmed5 & 1,31 & 0,01 & 0,02 & 0,00 & & 0,00 & 0,04 \\
\hline Pmed6 & 0,05 & 0,18 & 0,08 & 0,00 & 0,00 & 0,00 & 0,11 \\
\hline Pmed7 & 0,85 & 0,16 & 0,12 & 0,01 & 1,09 & 0,00 & 0,19 \\
\hline Pmed8 & 10,70 & 0,27 & 0,13 & 0,22 & & 0,01 & 0,21 \\
\hline Pmed9 & 2,99 & 1,31 & 0,46 & 0,26 & & 0,00 & 1,24 \\
\hline Pmed10 & 10,48 & 1,08 & 2,16 & 1,47 & & 0,03 & 1,45 \\
\hline Pmed11 & 0,53 & 0,18 & 0,24 & 0,47 & 0,53 & 0,00 & 0,33 \\
\hline Pmed12 & 68,39 & 0,65 & 0,17 & 1,36 & 0,29 & 0,02 & 0,56 \\
\hline Pmed13 & 6,26 & 1,23 & 17,49 & 0,16 & & 0,01 & 1,20 \\
\hline Pmed14 & 19,89 & 8,48 & 12,56 & 4,66 & & 0,14 & 2,09 \\
\hline Pmed15 & 66,01 & 6,70 & 0,93 & 0,76 & & 0,04 & 3,69 \\
\hline Pmed16 & 0,25 & 0,01 & 0,01 & 0,00 & 0,16 & 0,00 & 0,51 \\
\hline Pmed17 & 17,13 & 3,12 & 0,18 & 0,03 & 0,70 & 0,01 & 0,96 \\
\hline Pmed18 & 6,56 & 14,35 & 2,32 & 0,00 & & 0,15 & 1,63 \\
\hline Pmed19 & 53,73 & 36,01 & 2,63 & 14,53 & & 0,07 & 2,04 \\
\hline Pmed20 & 75,78 & 26,23 & 17,86 & 6,27 & & 0,09 & 4,33 \\
\hline Pmed21 & 0,35 & 0,15 & 0,01 & 0,00 & 0,19 & 0,00 & 0,86 \\
\hline Pmed22 & 13,41 & 9,92 & 5,57 & 4,29 & 5,41 & 0,19 & 1,44 \\
\hline Pmed23 & 10,50 & 13,50 & 1,77 & 1,00 & & 0,23 & 2,01 \\
\hline Pmed24 & 1,45 & 31,74 & 29,58 & 3,30 & & 0,08 & 2,59 \\
\hline Pmed25 & 3,65 & 21,08 & 0,04 & 27,21 & & 0,38 & 2,44 \\
\hline Pmed26 & 0,07 & 0,07 & 0,27 & 0,01 & 0,30 & 0,00 & 1,02 \\
\hline Pmed27 & 9,42 & 0,61 & 0,04 & 0,10 & 5,24 & 0,01 & 1,47 \\
\hline Pmed28 & 27,67 & 3,01 & 91,77 & 10,56 & & 0,05 & 2,73 \\
\hline Pmed29 & 1,12 & 91,49 & 0,01 & 18,15 & & 1,14 & 2,04 \\
\hline Pmed30 & 100,29 & 23,63 & 0,01 & 10,16 & & 0,35 & 4,98 \\
\hline Pmed31 & 4,15 & 0,07 & 0,03 & 0,02 & 0,15 & 0,00 & 0,81 \\
\hline Pmed32 & 14,09 & 19,83 & 3,25 & 10,15 & 1,43 & 0,06 & 2,12 \\
\hline Pmed33 & 59,70 & 96,81 & 58,98 & 11,53 & & 0,72 & 2,06 \\
\hline Pmed34 & 6,33 & 19,22 & 0,01 & 9,70 & & 0,39 & 3,68 \\
\hline Pmed35 & 1,87 & 0,80 & 0,22 & 1,58 & 0,33 & 0,01 & 1,34 \\
\hline Pmed36 & 9,89 & 12,72 & 7,07 & 6,07 & 1,76 & 0,08 & 2,98 \\
\hline Pmed37 & 9,38 & 143,74 & 0,04 & 11,88 & & 0,12 & 2,77 \\
\hline Pmed38 & 0,42 & 0,23 & 0,05 & 0,10 & 0,75 & 0,00 & 0,18 \\
\hline Pmed39 & 155,73 & 0,72 & 168,12 & 0,66 & 2,37 & 0,01 & 1,29 \\
\hline Pmed40 & 1,26 & 59,25 & 0,01 & 5,73 & & 0,19 & 2,06 \\
\hline
\end{tabular}

the stopping criterion is taken as the maximum allowed CPU time (second) as in $[8,10,11]$ and is set to one-tenth of the number of customers (i.e., $n / 10$ ) for each problem. The parameters of the proposed algorithm have a remarkable effect on the quality and effectiveness of the algorithm. Therefore, with the help of the initial experiments, the search range is taken as $[-10,10]$ and the population size and trial are set to 100 and 50, respectively, for all benchmarks.
The computational results for the benchmark problems are summarized in Table 1. In Table 1, the first column shows the problem name. The second and third columns denote the number of customers and the number of centers, respectively. The next six columns show the results from M-I, VNS, TS1 and TS-2 [9], SS [10], and BCOi [11], and the last column represents the results with the M-ABC algorithm. In Table 1, the best result achieved from 10 consecutive runs is reported 
for each test problems similar to [9-11] and bold values denote best-known solutions found with the M-ABC algorithm. The ratio of the number of problems where the best solution is achieved, to the total number of problems solved is given in the last row of Table 1 for each algorithm.

As seen from Table 1, the M-ABC algorithm finds the best-known solutions for 37 out of 40 test problems. Results in Table 1 show that M-ABC, VNS, and TS-2 perform similarly in terms of solution quality. Furthermore, better results are obtained with $\mathrm{M}-\mathrm{ABC}$ for pmed18 and pmed39, when compared to TS-2. M-ABC outperforms VNS for pmed39. It can be concluded from Table 1 that $\mathrm{M}-\mathrm{ABC}$ is better than M-I, TS-1, and BCOi on most of the test problems. BCOi's performance is superior to that of M-ABC only on pmed30, where BCOi approach is the best performer among all algorithms. For the small size problems $(p \leq 10)$ SS and M$A B C$ yield the same values in terms of the best value; however it would not be a fair comparison since SS was not applied to bigger problems where $p>10$ in [11]. In general, the results indicate that the $\mathrm{M}-\mathrm{ABC}$ algorithm is superior to traditional and new metaheuristics.

For a better evaluation of metaheuristic algorithms, not only the solution quality but also the computation times should be investigated. However, it is not very easy to make an objective comparison between metaheuristics because both the programming languages and the machine configurations are not generally comparable, and in most studies, the complexities of the algorithms are not reported. Nevertheless, an approximate comparison can be made based on the million floating point operations per second (MFLOP) values of the processors on which the algorithms were coded and run [43].

The heuristics proposed in [8] was implemented on a Sun SPARCstation 10, and the SS algorithm in [10] was executed on an Intel Pentium III processor operating at $300 \mathrm{MHz}$. Moreover, both heuristics were coded in Pascal with the Delphi 5.0 compiler. BCOi in [11] was implemented on an Intel Core 2 Duo E6750 processor and was coded in the $\mathrm{C}$ programming language with the $\mathrm{g}++$ compiler. The MFLOP values of the processor speeds based on the benchmark values obtained from the site http://www .netlib.org/benchmark/linpackjava/timings_list.html were used to normalize the CPU times. The MFLOP values and corresponding normalized $\mathrm{CPU}$ times spent on the benchmark problems for the algorithms are summarized in Table 2.

Table 2 shows the normalized CPU times with respect to the fastest CPU (Intel Core 2 Duo E6750) as the baseline. In Table 2, the CPU time in seconds spent by the M-ABC algorithm to achieve its best solution is reported like in $[8,10,11]$. Since M-ABC does not utilize any constructive initialization mechanism, it has a slower performance on the majority of small size test problems $(p \leq 10)$. On the other hand, M-ABC outperforms M-I, VNS, TS-1, and TS- 2 on most of the test problems having $p>10$ in terms of CPU time. As can be seen in Table 2, M-ABC is slightly slower than $\mathrm{BCOi}$, which is the quickest algorithm in test algorithms.

\section{Conclusion and Future Work}

In this paper, a modified artificial bee colony (M-ABC) algorithm is presented to solve $p$-center problems. The proposed approach has two main contributions: random key-based encoding for solution representation and a new multisearch strategy in which different search strategies are employed in one overall search process. The $\mathrm{M}-\mathrm{ABC}$ algorithm is compared to state-of-the-art metaheuristic algorithms with benchmark problems and is found to be effective and better than other algorithms in terms of both solution quality and $\mathrm{CPU}$ time on most of the benchmarks. The proposed M-ABC algorithm achieves best-known solutions for 37 out of 40 of the benchmark problems with competitive CPU times when compared to other metaheuristic algorithms. Future studies may include using adaptive selection for search strategies instead of random selection and application of the proposed approach on other real-life problems, such as vehicle routing or scheduling problems.

\section{Conflict of Interests}

The authors declare that there is no conflict of interests regarding the publication of this paper.

\section{References}

[1] D. Chen and R. Chen, "New relaxation-based algorithms for the optimal solution of the continuous and discrete p-center problems," Computers and Operations Research, vol. 36, no. 5, pp. 1646-1655, 2009.

[2] M. R. Garey and D. S. Johnson, Computers and Intractability: A Guide to the Theory of NP-Completeness, W. H. Freeman, New York, NY, USA, 1st edition, 1979.

[3] B. Pelegrin, "Heuristic methods for the p-center problem," RAIRO Recherche Operationelle, vol. 25, pp. 65-72, 1991.

[4] Z. Drezner, "The p-center problem: heuristic and optimal algorithms," Journal of the Operational Research Society, vol. 35, no. 8, pp. 741-748, 1984.

[5] R. Chandrasekaran and A. Tamir, "Polynomially bounded algorithms for locating p-centers on a tree," Mathematical Programming, vol. 22, no. 1, pp. 304-315, 1982.

[6] M. S. Daskin, Network and Discrete Location: Models, Algorithms, and Applications, John Wiley \& Sons, New York, NY, USA, 2011.

[7] G. Y. Handler, "P-center problems," in Discrete Location Theory, P. B. Mirchandani and R. L. Francis, Eds., pp. 305-347, John Wiley \& Sons, NewYork, NY, USA, 1990.

[8] N. Mladenović, M. Labbé, and P. Hansen, "Solving the p-center problem with Tabu search and variable neighborhood search," Networks, vol. 42, no. 1, pp. 48-64, 2003.

[9] C. Caruso, A. Colorni, and L. Aloi, "Dominant, an algorithm for the p-center problem," European Journal of Operational Research, vol. 149, no. 1, pp. 53-64, 2003.

[10] J. A. Pacheco and S. Casado, "Solving two location models with few facilities by using a hybrid heuristic: a real health resources case," Computers and Operations Research, vol. 32, no. 12, pp. 3075-3091, 2005. 
[11] T. Davidović, D. Ramljak, M. Šelmić, and D. Teodorović, "Bee colony optimization for the p-center problem," Computers and Operations Research, vol. 38, no. 10, pp. 1367-1376, 2011.

[12] D. Karaboga, "An idea based on honey bee swarm for numerical optimization," Tech. Rep. TR06, Erciyes University Press, Erciyes, Turkey, 2005.

[13] D. Karaboga and B. Basturk, "On the performance of artificial bee colony (ABC) algorithm," Applied Soft Computing Journal, vol. 8, no. 1, pp. 687-697, 2008.

[14] D. Karaboga and B. Basturk, "A powerful and efficient algorithm for numerical function optimization: artificial bee colony (ABC) algorithm," Journal of Global Optimization, vol. 39, no. 3, pp. 459-471, 2007.

[15] G. Zhu and S. Kwong, "Gbest-guided artificial bee colony algorithm for numerical function optimization," Applied Mathematics and Computation, vol. 217, no. 7, pp. 3166-3173, 2010.

[16] W.-F. Gao and S.-Y. Liu, "A modified artificial bee colony algorithm," Computers and Operations Research, vol. 39, no. 3, pp. 687-697, 2012.

[17] W. Gao and S. Liu, "Improved artificial bee colony algorithm for global optimization," Information Processing Letters, vol. 111, no. 17, pp. 871-882, 2011.

[18] G. Li, P. Niu, and X. Xiao, "Development and investigation of efficient artificial bee colony algorithm for numerical function optimization," Applied Soft Computing Journal, vol. 12, no. 1, pp. 320-332, 2012.

[19] F. Kang, J. Li, and Z. Ma, "Rosenbrock artificial bee colony algorithm for accurate global optimization of numerical functions," Information Sciences, vol. 181, no. 16, pp. 3508-3531, 2011.

[20] A. Singh, "An artificial bee colony algorithm for the leafconstrained minimum spanning tree problem," Applied Soft Computing Journal, vol. 9, no. 2, pp. 625-631, 2009.

[21] B. Alatas, "Chaotic bee colony algorithms for global numerical optimization," Expert Systems with Applications, vol. 37, no. 8, pp. 5682-5687, 2010.

[22] W. F. Gao, S. Y. Liu, and L. L. Huang, "A novel artificial bee colony algorithm with Powell's method," Applied Soft Computing, vol. 13, no. 9, pp. 3763-3775, 2013.

[23] Y. Xu, P. Fan, and L. Yuan, "A simple and efficient artificial bee colony algorithm," Mathematical Problems in Engineering, vol. 2013, Article ID 526315, 9 pages, 2013.

[24] B. Akay and D. Karaboga, "A modified Artificial Bee Colony algorithm for real-parameter optimization," Information Sciences, vol. 192, pp. 120-142, 2012.

[25] M. H. Kashan, N. Nahavandi, and A. H. Kashan, "DisABC: a new artificial bee colony algorithm for binary optimization," Applied Soft Computing Journal, vol. 12, no. 1, pp. 342-352, 2012.

[26] W. Y. Szeto, Y. Wu, and S. C. Ho, "An artificial bee colony algorithm for the capacitated vehicle routing problem," European Journal of Operational Research, vol. 215, no. 1, pp. 126-135, 2011.

[27] M. S. Uzer, N. Yilmaz, and O. Inan, "Feature selection method based on artificial bee colony algorithm and support vector machines for medical datasets classification," The Scientific World Journal, vol. 2013, Article ID 419187, 10 pages, 2013.

[28] A. Alvarado-Iniesta, J. L. Garcia-Alcaraz, M. I. RodriguezBorbon, and A. Maldonado, "Optimization of the material flow in a manufacturing plant by use of artificial bee colony algorithm," Expert Systems with Applications, vol. 40, no. 12, pp. 4785-4790, 2013.
[29] J. Ji, H. Wei, C. Liu, and B. Yin, "Artificial bee colony algorithm merged with pheromone communication mechanism for the 0 1 multidimensional knapsack problem," Mathematical Problems in Engineering, vol. 2013, Article ID 676275, 13 pages, 2013.

[30] Q.-K. Pan, M. Fatih Tasgetiren, P. N. Suganthan, and T. J. Chua, "A discrete artificial bee colony algorithm for the lot-streaming flow shop scheduling problem," Information Sciences, vol. 181, no. 12, pp. 2455-2468, 2011.

[31] L. Wang, G. Zhou, Y. Xu, S. Wang, and M. Liu, "An effective artificial bee colony algorithm for the flexible job-shop scheduling problem," The International Journal of Advanced Manufacturing Technology, vol. 60, no. 1-4, pp. 303-315, 2012.

[32] M. F. Tasgetiren, Q.-K. Pan, P. N. Suganthan, and A. H.-L. Chen, "A discrete artificial bee colony algorithm for the total flowtime minimization in permutation flow shops," Information Sciences, vol. 181, no. 16, pp. 3459-3475, 2011.

[33] M. Fatih Tasgetiren, Q. K. Pan, P. N. Suganthan, and A. Oner, "A discrete artificial bee colony algorithm for the noidle permutation flowshop scheduling problem with the total tardiness criterion," Applied Mathematical Modelling, vol. 37, no. 10-11, pp. 6758-6779, 2013.

[34] D. Karaboga, B. Gorkemli, C. Ozturk, and N. Karaboga, "A comprehensive survey: artificial bee colony $(\mathrm{ABC})$ algorithm and applications," Artificial Intelligence Review, pp. 1-37, 2012.

[35] J. C. Bean, "Genetic algorithms and random keys for sequencing and optimization," ORSA Journal on Computing, vol. 6, no. 2, pp. 154-160, 1994.

[36] L. V. Snyder and M. S. Daskin, "A random-key genetic algorithm for the generalized traveling salesman problem," European Journal of Operational Research, vol. 174, no. 1, pp. 38-53, 2006.

[37] J. J. M. Mendes, J. F. Gonçalves, and M. G. Resende, "A random key based genetic algorithm for the resource constrained project scheduling problem," Computers and Operations Research, vol. 36, no. 1, pp. 92-109, 2009.

[38] T. F. Noronha, M. G. Resende, and C. C. Ribeiro, "A biased random-key genetic algorithm for routing and wavelength assignment," Journal of Global Optimization, vol. 50, no. 3, pp. 503-518, 2011.

[39] P.-C. Chang, S.-H. Chen, and C.-Y. Fan, "A hybrid electromagnetism-like algorithm for single machine scheduling problem," Expert Systems with Applications, vol. 36, no. 2, pp. 12591267, 2009.

[40] A. Yurtkuran and E. Emel, "A new hybrid electromagnetismlike algorithm for capacitated vehicle routing problems," Expert Systems with Applications, vol. 37, no. 4, pp. 3427-3433, 2010.

[41] J. F. Gonçalves and M. G. Resende, "A parallel multi-population biased random-key genetic algorithm for a container loading problem," Computers and Operations Research, vol. 39, no. 2, pp. 179-190, 2012.

[42] J. E. Beasley, "A note on solving large p-median problems," European Journal of Operational Research, vol. 21, no. 2, pp. 270273, 1985.

[43] J. J. Dongarra, "Performance of various computers using standard linear equations software," ACM SIGARCH Computer Architecture News, vol. 20, no. 3, pp. 22-44, 1992. 


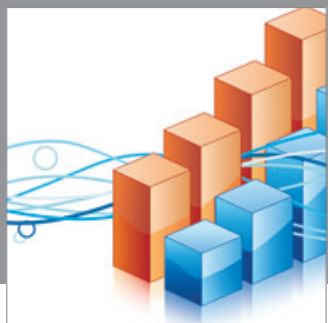

Advances in

Operations Research

mansans

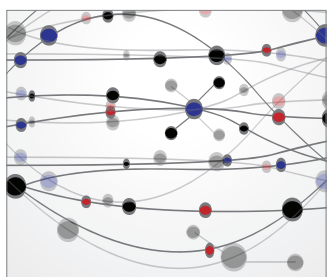

The Scientific World Journal
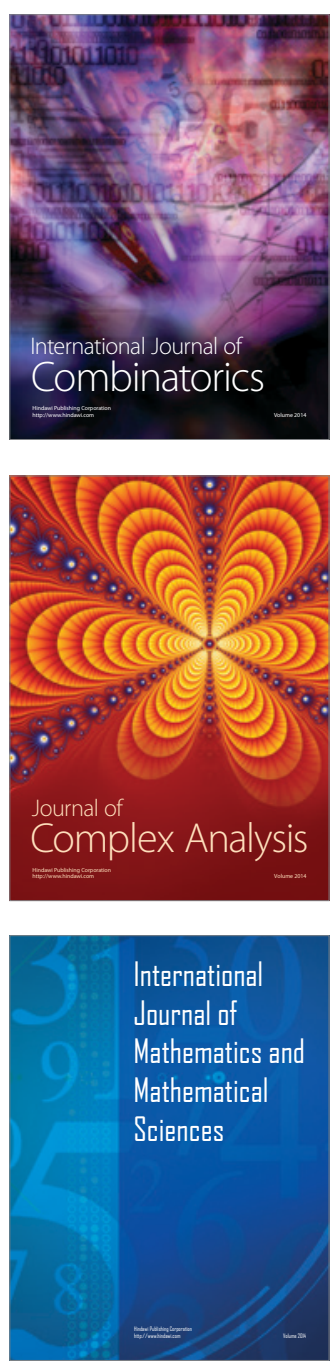
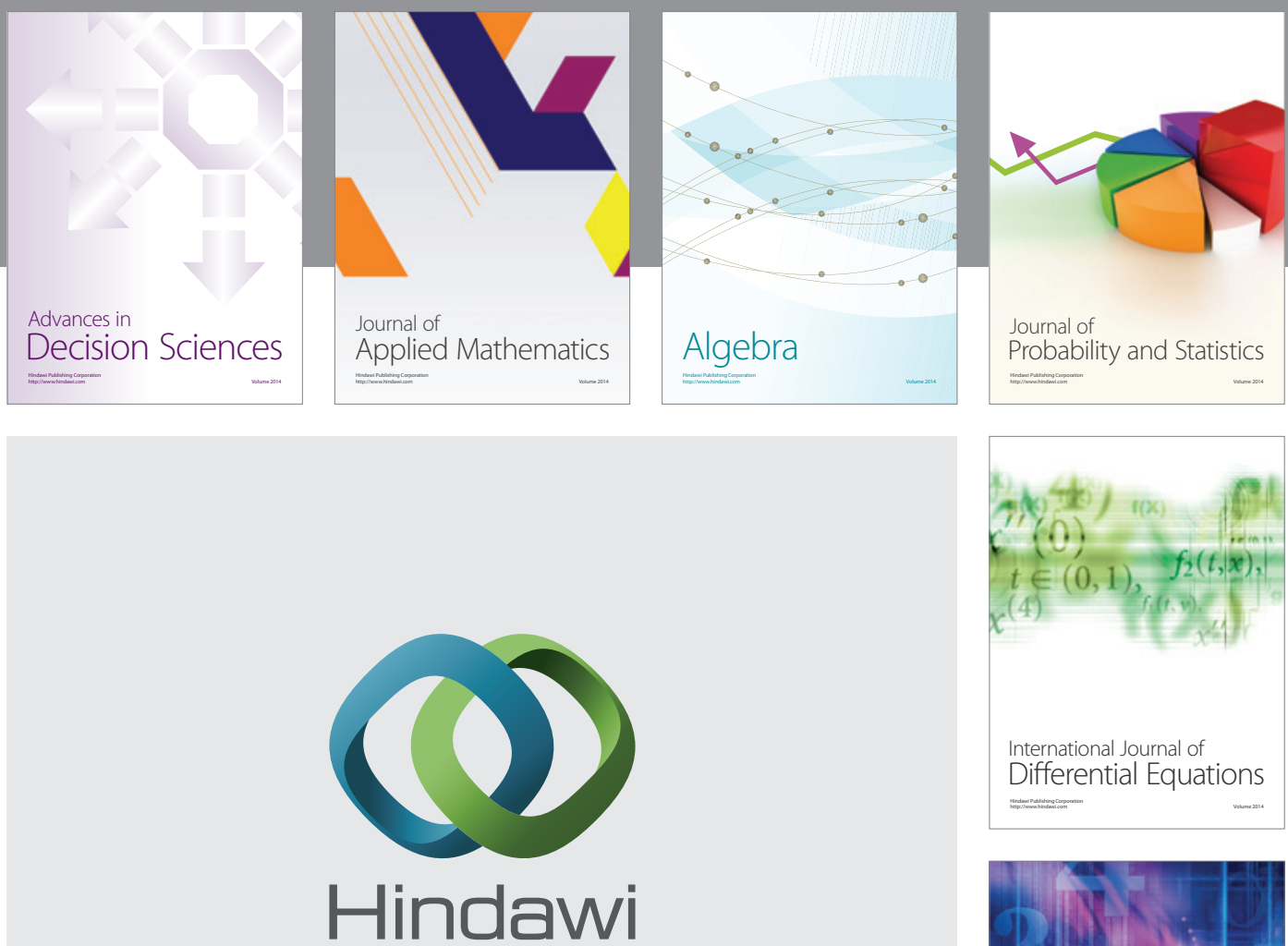

Submit your manuscripts at http://www.hindawi.com
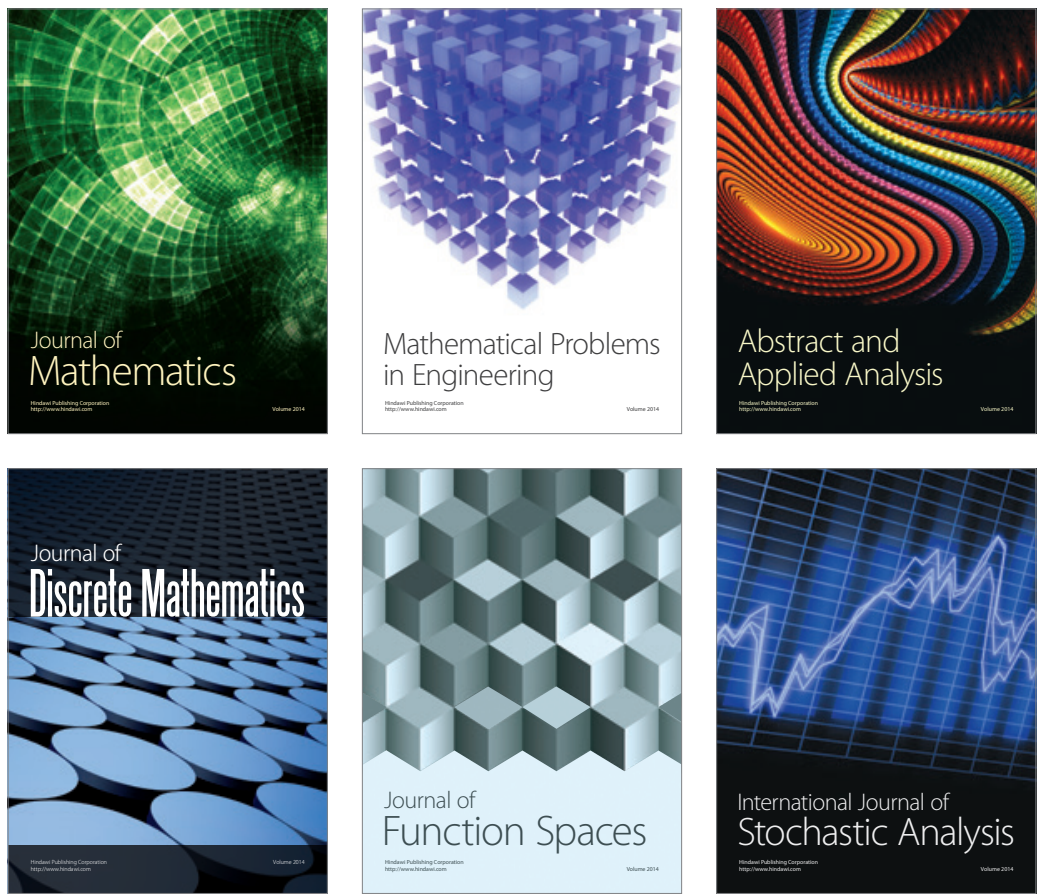

Journal of

Function Spaces

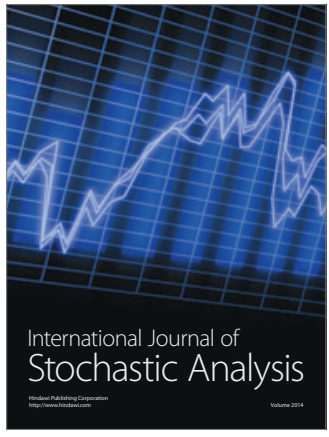

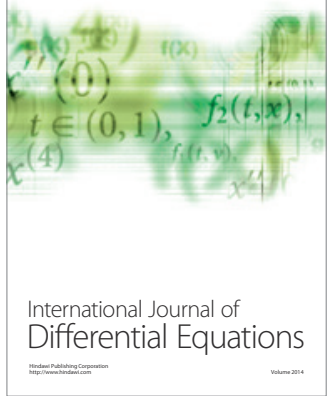
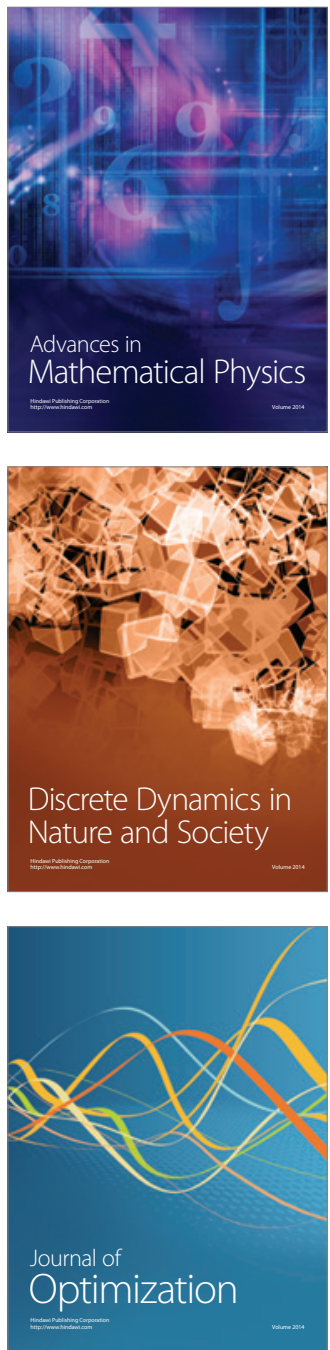